GRIMMELSHAUSEN - GESAMMELTE WERKE

\title{
IN EINZELAUSGABEN
}

\author{
Unter Mitarbeit von \\ Wolfgang Bender und Franz Günter Sieveke \\ herausgegeben von Rolf Tarot
}





\title{
GRIMMELSHAUSEN \\ Deß Weltberuffenen Simplicissimi Pralerey und Gepräng mit seinem Teutschen Michel
}

\author{
Herausgegeben von \\ Rolf Tarot
}

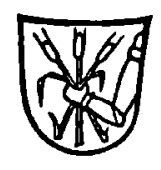

Max Niemeyer Verlag Tübingen 1976 
Abdruck der Erstausgabe von 1673

Mit 2 Abbildungen im Text

und 4 Abbildungen auf Tafeln

CIP-Kurztitelaufnahme der Deutschen Bibliothek

Grimmelshausen , Hans Jakob Christoffel von

[Sammlung]

Gesammelte Werke in Einzelausgaben / unter Mitarb. von Wolfgang Bender u. Franz Günter Sieveke hrsg. von Rolf Tarot. Tübingen : Niemeyer.

$\rightarrow$ Grimmelshausen, Hans Jakob Christoffel von : Dess

Weltberuffenen Simplicissimi Pralerey und Gepräng mit seinem

teutschen Michel

\section{Grimmelshausen, Hans Jakob Christoffel von}

Dess Weltberuffenen Simplicissimi Pralerey und Geprāng mit seinem teutschen Michel / hrsg. von Rolf Tarot. - 1. Aufl., Abdr. d.

Erstausg. von 1673. - Tübingen : Niemeyer, 1976.

(Gesammelte Werke in Einzelausgaben / Grimmelshausen)

ISBN 3-484-10264-0 kart.

ISBN 3-484-10263-2 Lw.

Gebundene Ausgabe: ISBN 3-484-10263-2

Broschierte Auggabo: ISBN 3-484-10264-0

(C)

Max Niemeyer Verlag Tübingen 1976

Alle Rechte vorbehalten - Printed in Germany

Satz und Druck P. Christian KG, Horb

Einband von Heinr. Koch Tübingen 